\title{
RELATIONSHIPS BETWEEN VARIATION OF NOCTURNAL DIPPING BLOOD PRESSURE AND CHRONOTYPES
}

\author{
*Felipe Leonardo and Débora Aparecida Rodrigueiro
}

Rua Cláudio Manoel da Costa, 202. Ap. 12 - Jd. Vergueiro - Sorocaba/SP - CEP: 18030-083

\section{ARTICLE INFO}

\section{Article History:}

Received $14^{\text {th }}$ March, 2020

Received in revised form

$26^{\text {th }}$ April, 2020

Accepted $09^{\text {th }}$ May, 2020

Published online $29^{\text {th }}$ June, 2020

\section{Key Words:}

Fenômenos cronobiológicos; Ritmo circadiano; Hipertensão Arterial Sistêmica;

Monitorização Ambulatorial da

Pressão Arterial.

*Corresponding author: Felipe Leonardo,

\begin{abstract}
A Chronobiology, a science that studies the temporal organization of living beings, establishes molecular mechanisms that explain the rhythmicity observed in organisms. Between the rhythmic variations observed is blood pressure, which shows a morning rise and nocturnal dipping, the latter being important in predicting cardiovascular risk. The individuals can also be chronophenotypically classified according to their daily preferences, in morningness-eveningness. Objectives: To verify the hypothesis of a relationship between the morning chronotype and the nondipper night pressure profile. Methodology: Descriptive cross-sectional study, with the realization of the noninvasive ambulatory monitoring of blood pressure to obtain the pressure curve and answer the questionnaire of Ostberg's personal preferences. Results: The survey included twenty participants, from nine were classified as dipper and eleven as nondipper; in addition, twelve were classified as morning and eight as non-morning. The hypothesis test revealed statistical significance in the relationship between morning chronotype and nocturnal non-descent pattern of blood pressure $(p=0.0493)$, confirming the initial hypothesis, which implies greater risk cardiovascular to this population. Conclusion: The relationship between morning and morning non-nocturnal blood pressure decrease, and further studies are needed to better characterizing this relationship.
\end{abstract}

Copyright (C) 2020, Felipe Leonardo and Débora Aparecida Rodrigueiro. This is an open access article distributed under the Creative Commons Attribution License, which permits unrestricted use, distribution, and reproduction in any medium, provided the original work is properly cited.

Citation: Felipe Leonardo and Débora Aparecida Rodrigueiro. "Relationships between variation of nocturnal dipping blood pressure and chronotypes", International Journal of Development Research, 10, (06), 36796-36801.

\section{INTRODUÇÃO}

A cronobiologia é um ramo da ciência que estuda a organização temporal dos seres vivos, tendo-se oficializado como disciplina científica em 1960 após o Cold Spring Harbor Symposium on Biological Clock, ocorrido em Nova Iorque (Araujo \& Marques, 2002). Apesar disso, a descoberta da existência de ritmos biológicos endógenos já vem sendo estudada desde 1729, quando um astrônomo francês, J.J. De Mairan percebeu que os movimentos das folhas da planta Mimosa pudica obedeciam um ritmo de aproximadamente 24 horas mesmo isolada da variação exógena de claro e escuro (Volkov et al., 2011). O ambiente apresenta diversos ciclos geofísicos e astronômicos que agem de forma rítmica, como por exemplo, o ritmo claro-escuro, que depende do movimento de rotação da terra sobre seu eixo; o ritmo das estações do ano e suas características, ditado pela translação da Terra em torno do centro do Sistema Solar; entre outros (Foster \& Roenneberg, 2008).
Os organismos que se adaptarem a tal ritmicidade garantem vantagem sobre os demais, sendo então selecionados para transmitir seus genes para a próxima geração. A necessidade de conformação do ritmo endógeno aos ciclos externos foi um determinante para o estabelecimento da fisiologia dinâmica de organismos de praticamente todos os níveis taxonômicos atuais. Estes conseguiram ser beneficiados pela variação claroescuro, quente-frio, seco-úmido e tantas outras variáveis que dominavam o ambiente (Jansen et al., 2007). Evolutivamente, os seres vivos desenvolveram a capacidade de variar seu ritmo interno para enfrentar as alterações externas de forma independente das mesmas, mecanismo esse conhecido como antecipação. Dessa forma não é mais necessária uma resposta à mudança, pois ela acontece antes, preparando o organismo para a mesma. Assim surgiu a ideia de um ritmo endógeno ajustado ao ambiental (Acúrcio \& Rodrigues, 2009; Jansen et al., 2007) São conhecidos diversos ritmos biológicos, dentre eles: circadiano (cerca de um dia, com período de $24 \pm 4 \mathrm{~h}$ ); ultradianos (com período de menos de 20h) e infradianos (com período de mais de $28 \mathrm{~h}$ ), no qual se encaixam o ritmo 
circanual (cerca de um ano), circalunar (cerca de uma lunação, ou 30 dias) e circasseptano (cerca de uma semana). Aquele com expressão mais significativa e com maior ênfase de estudos é o ritmo circadiano, que é o ritmo também das variações atividade-repouso e sono-vigília (Aschoff, 1979). Os mecanismos moleculares do ritmo circadiano já estão completamente elucidados (Jansen et al., 2007; Pereira et al., 2009), e estão envolvidos nele cinco genes: Cry (Cryptochrome), Per (Period), Tim (Timeless), Clk (Clock) e Cyk (Cycle) sendo que os quatro últimos funcionam em pares. $\mathrm{O}$ gene Cry expressa um fotorreceptor sensível à luz azul de mesmo nome, dando início à cascata molecular simplificada que segue (Kidd et al., 2015): os RNA mensageiros dos genes $\mathrm{Clk} / \mathrm{Cyk}$ dão origem às proteínas de mesmo nome que se acoplam formando a proteína CLK-CYK. Esta adentra o núcleo da célula ativando a região promotora dos genes Tim/Per. Então o RNA mensageiro transcrito dá origem às proteínas de mesmo nome. Com o passar do tempo elas se acumulam e acabam por acoplar-se, formando a proteína TIMPER. Essa nova proteína entra no núcleo celular e impede a ativação realizada pela proteína CLK-CYK sobre o gene promotor da síntese delas mesmas, agindo como um sistema de retroalimentação negativa. Depois de um tempo, elas são degradadas espontaneamente, podendo haver nova ativação do promotor e reiniciando o ciclo. Desse mecanismo, obtém-se a existência de um ritmo interno com duração aproximada de 25 horas, ditado pelo relógio biológico localizado no núcleo supraquiasmático (NSQ) do hipotálamo, que trabalha simultaneamente com outros relógios biológicos ainda não completamente elucidados, contando ainda com o ajuste de um fator externo (Aschoff, 1979; Jansen et al., 2007).

São chamados de zeitgeber (do alemão, "aquele que impõe o tempo") os fatores exógenos que têm a capacidade de arrastamento, ou seja, adiantar ou atrasar a fase de um ciclo para que ele se alinhe com a fase do ciclo exógeno. Dentre eles o mais conhecido é a luz (nos animais diurnos) que causa uma dessincronização forçada do relógio biológico ao estimular receptores na retina contendo melanopsina, que sinaliza pelo feixe retino-hipotalâmico, até alterar o sistema de retroalimentação genética (Cry-Per-Tim-Clk-Cyk), levando a uma ressincronização, ou seja, alinhando as fases dos dois ciclos (externo e interno). Ficou evidente pelos últimos estudos que o principal neurotransmissor envolvido nesse processo de reorganização do ritmo circadiano é o peptídeo vasoativo intestinal (VIP) (An et al., 2013). Isso fica comprovado quando se deixa um animal em livre curso, afastado de qualquer zeitgeber, pois estes passam a assumir seu ritmo interno, desalinhando-se do ciclo claro-escuro ambiental (Aschoff \& Pohl, 1978; Aschoff, 1979; Jansen et al., 2007).

Com o ritmo estabelecido, o NSQ é capaz de sincronizar a periodicidade de muitas funções vitais a partir de suas eferências, principalmente: centros hipotalâmicos facilitadores de comportamento ligados à atividade/inatividade e neuroendócrinos responsáveis pela secreção de hormônios, além de neurônios hipotalâmicos pré-autonômicos que afetam centros simpáticos ou parassimpáticos no tronco cerebral ou medula espinhal. Assim, muitas atividades ganham uma dinamicidade de função pré-estabelecida, ou um ritmo, como a freqüência cardíaca, temperatura corporal, pressão arterial, nível plasmático de cortisol, somatotrofina, nível de excreção de potássio, entre outros (Aloe et al., 2005; González \& AstonJones, 2006). A pressão arterial (PA) de um indivíduo tem um ritmo circadiano interno, atingindo seu nadir (menor valor) à noite, enquanto o indivíduo está dormindo. Ela aumenta potencialmente nas primeiras horas da manhã, atingindo sua acrofase (maior valor) algumas horas depois de acordar. Assim se mantém até o entardecer, onde começa a baixar. Muitos são os fatores que causam oscilação da PA ao longo do dia: consumo de álcool e cafeína, atividade física, ciclo sono/vigília, estado emocional e temperatura ambiental são exemplos destes contribuintes externos. (Flores, 2011) Conhecendo a enorme influência do sistema nervoso autônomo sobre a regulação da PA é justificada a ritmicidade que ela segue, pois o tônus simpático é dominante durante o período de atividade diurno e o tônus vagal (parassimpático) durante o período noturno de sono. Da mesma forma outros reguladores endógenos - de mesma variação circadiana quando mensurados no plasma - contribuem para a sua formação no repouso, como o cortisol, renina, angiotensina I, angiotensina II, aldosterona e peptídio natriurético atrial (Hermida et al., 2007). Esses e outros fatores endoteliais responsáveis pela regulação pressórica demonstraram-se cronobiologicamente influenciáveis, tendo relação inclusive com a fisiopatologia de alguns distúrbios cardiovasculares (Tobaldini et al., 2019). Esse descenso noturno por volta de 10 a $20 \%$ da média diária é considerado um parâmetro para classificar as pessoas em quatro perfis diferentes, de acordo com seu valor de PA enquanto dormem: extreme-dippers (descenso $\geq 20 \%$ ), dippers (descenso $\geq 10 \%$ ), non-dippers (descenso $<10 \%$ ) e inverse-dippers/risers (descenso $<0 \%$ ). Algumas causas são usadas para explicar esse fenômeno, como a disautonomia, incluindo aquela causada pelo diabetes, assim como ele já foi relacionado a condições como a insuficiência cardíaca, doença renal crônica, insônia familiar, disfunções endócrinas, sensibilidade anormal ao sódio, atividades noturnas e má qualidade do sono. Contudo, seu mecanismo ainda não está bem claro (Hermida et al., 2007).

Um estudo japonês demonstrou que um descenso de apenas $5 \%$ da pressão arterial sistólica em pacientes hipertensos está associado a um aumento do risco cardiovascular de $31 \%$ e que pacientes normotensos non-dippers tem o mesmo risco que pacientes hipertensos dippers, fato também evidenciado por outros estudos (Ohkubo et al., 2002; Pirsarabi et al., 2018; Verdecchia et al., 1993). Dessa forma, deve-se prestar atenção nesse fator ao considerar a terapêutica necessária, a fim de diminuir o risco cardiovascular, pois se sabe que a resposta de certos medicamentos anti-hipertensivos varia de acordo com a hora da sua administração, o que é chamado de cronofarmacologia. Uma pesquisa demonstrou associação na administração medicamentosa noturna, antes de se deitar, com um maior descenso noturno, assim aumentando a proteção cardiovascular de indivíduos non-dipper (Crespo et al., 2013).

Ainda dentro do contexto da cronobiologia, os humanos se caracterizam por variada preferência pelo melhor horário para realizar atividades cotidianas, como trabalhar, estudar, exercitar-se e até alimentar-se. São os chamados cronotipos. Àqueles com preferência diurna chamamos matutinos, os com preferência pelo período da tarde ou início da noite, vespertinos, e, ainda, àqueles que optam por entre esses dois turnos, intermediários (Baehr et al., 2000). Muitas são as pesquisas de cunho molecular e genético para elucidar o mecanismo dessa inclinação, a título de exemplo: polimorfismos nos genes Clock (CLK) e Period (PER) 1, 2 e 3. Ainda não obtiveram resultados conclusivos, mas confirmouse a idéia de que tais polimorfismos são capazes de alterar o comprimento do período endógeno, por alterarem a fase 
sincronizada pela luz (Pereira et al., 2009). Outrossim, também percebeu-se uma relação epidemiológica dos cronotipos com determinantes sociais como sexo e idade (Matchock, 2018; Paine et al., 2006). Alguns autores levantam o questionamento se hábitos, como dormir mais cedo no caso dos matutinos, são guiados pela regulação característica destes no processo circadiano do sono ou se não seria o último a ser construído com base nesses hábitos (Duffy et al., 2001). Apesar disso, estudos envolvendo a medição da temperatura central de forma contínua por 36 horas mostram que há uma diferença na variação do seu ciclo em indivíduos com diferentes cronotipos, sendo os matutinos caracterizados por terem um pico mais cedo que os vespertinos (Baehr et al., 2000; Taillard et al., 2003). Baseando-se nessa variabilidade cronotípica da temperatura corporal, que possui uma forte influência da regulação simpática (Irigoyen et al., 2001) - aventou-se a hipótese de que possa haver igual relação entre os cronotipos e a variação do ciclo da pressão arterial. De acordo com a variação observada nos indivíduos dos estudos citados, onde os matutinos possuem um pico de temperatura matinal antes dos vespertinos, pode coexistir a relação entre o perfil nondipper e os matutinos, pois estes apresentariam seu ciclo adiantado. O objetivo do presente estudo é analisar se essa relação se encontra factível na prática clínica, além de descrever cronofenotipicamente a amostra adquirida.

\section{MATERIAIS E MÉTODOS}

Esta pesquisa foi iniciada após aprovação no Comitê de Ética em Pesquisa da Faculdade de Ciências Médicas e da Saúde da Pontifícia Universidade Católica - Campus Sorocaba, fundado sob o CAAE 88246418.3.0000.5373 que deu seu parecer consubstanciado sob o registro 2.736.707. A casuística consistiu em uma amostra de pacientes que se submeteram regularmente ao exame do monitorização ambulatorial da pressão arterial (MAPA) no Conjunto Hospitalar de Sorocaba (CHS) nos meses de Agosto de 2018 a Janeiro de 2019, sendo sua maioria para investigação de hipertensão arterial. Consideramos como critérios de exclusão: trabalhadores com turno noturno e aqueles que realizaram viagens entre fusos nos últimos três meses. Após lerem e assinarem o Termo de Consentimento Livre e Esclarecido (TCLE), os voluntários responderam ao questionário sobre preferências pessoais (Horne \& Ostberg, 1976), por meio do qual foi possível classificá-los de acordo com seus cronotipos em matutinos, moderadamente matutinos, intermediários, moderadamente vespertinos ou vespertinos. Os questionários foram corrigidos duas vezes em dias diferentes, para reduzir as possibilidades de erro. Os resultados foram compilados em tabelas, para posterior comparação com os resultados do exame de MAPA. A aquisição dos dados pressóricos por meio do exame MAPA teve como base a pressão do período do dia e a do período da noite. A razão entre esses dois valores deu a proporção de descenso noturno (PDN), que pode ser analisada em relação à pressão arterial média. Eles foram tabelados para posterior comparação com as informações dos questionários preenchidos. Dessa forma o paciente foi classificado como dipper $(\mathrm{PDN} \geq 0,1)$ ou non-dippers $(\mathrm{PDN}<0,1)$. Na análise estatística investigamos a significância dos resultados dos grupos amostrais através do teste T de Student não pareado.

\section{RESULTADOS}

Ao final da aquisição de dados obtivemos resultados referentes a vinte e quatro pacientes que submeteram-se ao exame do
MAPA. Destes, um foi excluído por ter apresentado problema na coleta dos dados do MAPA, um por apresentar deficiência intelectual importante, que a incapacitou de responder os questionários e dois por trabalharem no turno noturno, chegando numa amostra válida final de vinte $(n=20)$. Dos 20 pacientes interrogados, 11 eram do sexo feminino $(55 \%)$ e nove do sexo masculino (45\%); dois tinham menos de 40 anos (10\%), oito tinham entre 41 e 59 anos $(40 \%)$ e dez tinham mais que 60 anos (50\%). A média de idade entre estes foi de 56 anos $\pm 13,4$ anos (26 a 72 anos). Para melhor categorizar os resultados, foi considerado um padrão dipping quando o valor superava $10 \%$ e non-dipping quando o valor era menor $10 \%$. A subclassificação riser (menor que $0 \%$ ) e extreme-dipper (maior que 20\%) não foi utilizada e foi incorporada nas categorizações anteriores. $\mathrm{Na}$ amostra selecionada, foram nove os pacientes categorizados como dipper (45\%) e 11 como nondipper (55\%). Para melhor analisar a amostra resolveu-se por unificar os subtipos matutino e moderado matutino na categoria matutino e os subtipos intermediário, moderado vespertino e vespertino na categoria não-matutino. $\mathrm{Na}$ amostra selecionada, foram 12 classificados como matutinos $(60 \%)$ e oito como não-matutinos (40\%). Foi verificado que dentre os matutinos, cinco eram dipper $(41,7 \%)$ e sete eram non-dipper (58,3\%), já entre os não-matutinos, quatro eram dipper $(50 \%)$ e quatro non-dipper (50\%). Essa associação se demonstrou significativa $(p=0,0493, I C=95 \%)$. As relações entre sexo e PDN, idade e PDN, sexo e cronotipo não se demonstraram estatisticamente significativa $(\mathrm{p}>0,05, \mathrm{IC}=95 \%)$, contudo a relação entre idade e cronotipo mostraram uma correlação. Para tal foi dicotomizada a distribuição etária entre menores de 60 anos e maiores ou iguais a 60 anos, havendo relação positiva entre idade maior que 60 anos e o cronotipo matutino $(p=0.037, I C=95 \%)$.

\section{DISCUSSÃO}

Ontogenética da distribuição dos cronotipos: A distribuição de cronotipos na amostra adquirida, a saber de $60 \%$ matutinos (profundos e moderados), 35\% intermediários e $5 \%$ vespertinos (moderados), apresentou significante variação daquelas observadas na literatura. Em 1976, Horne ao publicar a validação da escala de autoavaliação utilizada no presente estudo encontrou uma variação percentual de 37, 21 e 42\% (matutinos, intermediários e vespertinos, respectivamente); estudos mais recentes utilizando o mesmo questionário mostraram distribuição de $8-49 \%$ de matutinos, $45-71 \%$ de intermediários e 6-32\% de vespertinos (Horne \& Ostberg, 1976; Knutson \& von Schantz, 2018; Matchock, 2018; Paine et al., 2006; Roenneberg et al., 2003). Na amostra apresentada foi verificada a ausência de indivíduos profundamente vespertinos (tendo apenas um indivíduo moderamente vespertino nesse grupo), tendo, contudo prevalência dos cronotipos matutinos. A tendência à matutinidade encontrada pode ser explicada por fatores amostrais e geográficos. Este dado pode ser justificado pela faixa etária dos entrevistados, os quais $90 \%$ tinham mais de 40 anos, tendo este fator, inclusive, demonstrado relevância estatística na análise realizada. De fato, já ficou comprovado que os indivíduos tendem a se "matutinizar" com o passar dos anos e isso se deve a um avanço de fase, fenômeno cronobiológico que modifica de forma acentuada a expressão do sistema de temporização circadiana em humanos, alterando o cronofenótipo para um costume mais propenso ao despertar e ir se deitar mais cedo, entre outras características (Duarte, 2018). Entre os fatores que determinam um cronotipo, os zeitgebers estão entre os 
mais importantes, agindo tanto epigeneticamente quanto por pressão seletiva. A luz é o regulador circadiano mais estudado, sendo, portanto, a incidência solar (que é inversamente proporcional à latitude) uma variável a ser considerada ao analisar a distribuição cronofenotípica. Ela pode agir sobre a classificação de um cronotipo de forma a influenciar uma resposta tônica (causada por um fator permanente, e, por isso, sustentada longitudinalmente no tempo) ou uma resposta fásica (que vem em estações, não se sustentando longitudinalmente no tempo). A cidade de realização do estudo possui coordenada geográfica de latitude $23^{\circ} \mathrm{S}$, aproximadamente sobre o Trópico de Capricórnio, o que a caracteriza como uma região de intermediária incidência solar, não demandando resposta tônica à luz significante para afetar os dados (Díaz-Morales \& Sánchez-López, 2005; Duarte, 2018). Quando se introduz a variável longitude à análise, é possível incorporar a variação da incidência solar ao longo do ano, expressa como o fotoperíodo daquela região. Os meses de coleta de dados da nossa pesquisa foram de Agosto a Janeiro, que corresponde ao fim do inverno e período crescente de radiação solar caracterizado pela primavera e início do verão. Este fato isolado pode ser um fator distrativo durante $o$ preenchimento do questionário, ou resposta fásica à luz, tendendo seu respondente à matutinidade. Estes dados podem ser melhor esclarecidos por um modelo funcional multivariado que considera a diversidade de mecanismos fisiológicos de ajustes temporais (Duarte, 2018). Além de todos esses fatores mensuráveis, a distribuição dos cronotipos está subjugada a fatores sociais e culturais, que são específicos de uma localidade e até mesmo de uma família, o que nos leva a propor, conforme Duarte em seu livro sobre os cronotipos "que o cronotipo é uma propriedade intrínseca mutável e não uma propriedade característica do sistema circadiano humano" (Duarte, 2018).

Determinantes da depleção noturna da pressão arterial: Por mais que ainda sejam pouco compreendidos os mecanismos responsáveis pela depleção noturna da pressão arterial, a principal hipótese até o momento é a diminuição da atividade e tônus simpático no período de sono. Este fato é claramente demonstrado em pacientes com hipotensão ortostática, que possuem denervação simpática ou por pacientes com feocromocitoma, cujos níveis de catecolaminas permanecem altos durante à noite. Contudo, não se explica atualmente porque os demais pacientes não caracterizados acima possuem essa mesma não depleção (Pickering, 1990; Noll et al., 2001). Contudo, antes de se analisar os dados obtidos com essa pesquisa, é necessário levantar uma ressalva: a diminuída acurácia na aquisição fiel do perfil pressórico por meio da técnica do MAPA quando há uma perturbação no sono causada pela execução do exame noturno, o que aumentaria o número de falsos positivos (Dimsdale \& Heeren, 1998; Mochizuki et al., 1998; Noll et al., 2001). A ferramenta metodológica foi escolhida mesmo assim por ser a melhor dentre as disponíveis no local do estudo, tendo sido tentado reduzir esse viés de má qualidade crônica do sono por meio do critério de exclusão "problemas para dormir" no questionário, não tendo sido possível avaliar, contudo, a qualidade pontual do sono na noite do exame. A prevalência de cada padrão de descenso noturno, $45 \%$ sendo dipping's e 55\% sendo nondipping's, encontrada nesta pesquisa se aproxima razoavelmente do valor obtido de outros estudos, onde há uma razão de 47-83\% de dipping's e 17-53\% de non-dipping's, mostrando inclusive que há mais fatores envolvidos em sua modulação do que os critérios inclusivos e exclusivos que esses estudos foram capazes de abarcar (Mochizuki et al., 1998; O'Brien et al., 1988; Roman et al., 1997; Sierra et al., 2009).

O exame do MAPA hoje orienta a forma de tratamento e o risco cardiovascular do paciente hipertenso, pois que está amplamente evidente na literatura a relação entre a cronoterapia (relação do padrão de descenso pressórico com a hora de administração dos antihipertensivos, tendo os nondippers elevado benefício em tê-los administrados antes de dormir, em vez de tomar antes do café da manhã, como é convencionalmente feito) e o elevado risco cardiovascular, principalmente para hipertrofia do ventrículo esquerdo identificado em nondippers igualmente. Contudo deve-se prestar atenção à técnica de diagnóstico empregada ao determinar esse perfil pressórico noturno - fixo ou conforme o diário de atividades do paciente - pois o descenso está relacionado com o sono, e este não corresponde muitas vezes ao horário previamente determinado pelo aparelho, o que pode gerar falsos positivos (Hermida et al., 2008; Hermida et al., 2007; Roman et al., 1997). Há na literatura um estudo semelhante, porém com objetivos diferentes, que apresentou dados a respeito dos hábitos cronofenotípicos de sono e vigília e o descenso da pressão arterial, não tendo sido encontrado significância estatística nessa comparação, como foi o caso dos dados obtidos por essa pesquisa (Kani et al., 2015). Contudo, dois mecanismos indiretos nos ajudam a relacionar essas variáveis: o primeiro diz respeito às causas de aumento da atividade simpática durante o sono (como a insônia ou a apneia obstrutiva do sono, por exemplo), que pode também estar elevado no início da noite em indivíduos vespertinos e no fim da noite em indivíduos matutinos (Bansil et al., 2011); o segundo mecanismo, justificando o primeiro, diz respeito ao dado correlato de aumento da prevalência de matutinos e da prevalência de perfil nondipper entre os idosos, o que pode confirmar o dado encontrado de relação da matutinindade com a ausência de descenso (Duarte, 2018). Todavia, são necessários novos estudos com uma amostra maior para melhor relacionar esses dados.

\section{Conclusões}

A cronobiologia se apresenta como um ramo científico promissor para a personalização da clínica, aumentando a eficácia dos métodos já utilizados no diagnóstico e tratamento de agravos e patologias. Dentre os ritmos biológicos estudados nessa disciplina, destaca-se o circadiano, caracterizado pelo ciclo sono-vigília que influencia ademais em tantos outros circuitos fisiológicos, como é o caso da pressão arterial, que apresenta uma fase de descenso durante o sono, fase essa ausente em alguns pacientes hipertensos, denominados nondippers. Os indivíduos podem ainda ser classificados cronofenotipicamente como aqueles que preferem dormir e acordar mais tarde (vespertinos) ou mais cedo (matutinos), tendo alguns níveis intermediários entre esses, chamados cronotipos. A amostra apresentada caracterizou-se pela ausência de cronotipos vespertinos, o que prejudicou até certo ponto uma caracterização mais fidedigna e pode ser justificada pela prevalência de pessoas acima dos 40 anos, fase em que se inicia a matutinização do sistema de temporização circadiano interno. Não foram encontradas relações estatisticamente significantes entre sexo e cronotipo ou entre o sexo e o padrão de descenso noturno. Contudo, não se pode excluir tal relação, visto que a amostra apresentada foi pequena e não permitiu uma análise mais eficiente dos dados, sendo necessários estudos com amostra maior para analisar essas relações. 
Foi encontrada uma relação entre idade e cronotipo, assim como previsto pela literatura, entretanto não foi encontrada nenhuma relação entre idade e padrão de descenso noturno, como era esperado. Assim como dito anteriormente, são necessários estudos com amostra maior para verificar tais relações.Não obstante a já citada reduzida amostra, foi possível confirmar a hipótese principal de associação do cronotipo matutino com o padrão de descenso noturno nondipper. No entanto, visto que o exame de MAPA pode estar relacionado com uma especificidade duvidosa desta última variável, é necessário repensar as técnicas de purificar e analisar tal dado para que possa haver uma relação mais substancial dessa relação.

\section{REFERÊNCIAS}

Acúrcio AR, Rodrigues LM. 2009. Os Ritmos da Vida - Uma Visão Actualizada da Cronobiologia Aplicada. Rev. Lusófona de Ciências e Tecnologias da Saúde. 6(2): 21634.

Aloe F, Azevedo AP, Hasan R (2005). Mecanismos do ciclo sono-vigília. Rev Bras Psiquiatr. 27(1): 33-9.

An S, Harang R, Meeker K, Granados-Fuentes D, Tsai CA, Mazuski C, et al (2013). A neuropeptide speeds circadian entrainment by reducing intercellular synchrony. Proc Natl Acad Sci USA. 110(46): 4355-61.

Araujo JF, Marques N (2002). Cronobiologia: uma multidisciplinaridade necessária. Margem. 15: 95-112.

Aschoff J (1979). Circadian Rhythms: Influences of Internal and External Factors on the Period Measured in Constant Conditions. Zeitschrift Für Tierpsychologie. 49(3): 22549.

Aschoff J, Pohl H (1978). Phase Relations between a Circadian-Rhythm and Its Zeitgeber within Range of Entrainment. Naturwissenschaften. 65(2): 80-4.

Baehr EK, Revelle W, Eastman CI (2000). Individual differences in the phase and amplitude of the human circadian temperature rhythm: With an emphasis on morningness-eveningness. J Sleep Research. 9(2): 11727.

Bansil P, Kuklina EV, Merritt RK, Yoon PW (2011). Associations Between Sleep Disorders, Sleep Duration, Quality of Sleep, and Hypertension: Results From the National Health and Nutrition Examination Survey, 2005 to 2008. The Journal of Clinical Hypertension, 13(10), 739-43.

Crespo JJ, Piñeiro L, Otero A, Castiñeira C, Ríos MT, Regueiro A, et al (2013). Administration-time-dependent effects of hypertension treatment on ambulatory blood pressure in patients with chronic kidney disease. Chronobiology International. 30(1-2): 159-75.

Díaz-Morales JF, Sánchez-López MP (2005). Composite scales of morningness and preferences: Preliminary validity data in Peruvian undergraduates. Ergonomics. 48(4), 354-63.

Dimsdale JE, Heeren MM (1998). How Reliable Is Nighttime Blood Pressure Dipping? Am J Hypertens. 7061(98): 6069.

Duarte LL (2018). Cronotipos humanos. Editora UFRB, Cruz das almas, Brasil.

Duffy JF, Rimmer DW, Czeisler CA (2001). Association of intrinsic circadian period with morningness-eveningness, usual wake time, and circadian phase. Behavioral Neuroscience. 115(4): 895-9.
Flores Q (2011). Cronoterapêutica: uma abordagem no tratamento da hipertensão Cronoterapêutica: uma abordagem no tratamento da hipertensão. Rev Portug Farmacoterapia 1: 3-10.

Foster RG, Roenneberg T (2008). Human Responses to the Geophysical Daily, Annual and Lunar Cycles. Current Biology. 18(17): 784-94.

González MMC, Aston-Jones G (2006). Circadian regulation of arousal: Role of the noradrenergic locus coeruleus system and light exposure. Sleep. 29(10): 1327-36.

Hermida RC, Ayala DE, Calvo C (2008). Chronotherapy in Resistant Hypertension Chronotherapy Improves Blood Pressure Control and Reverts the Nondipper Pattern in Patients With. J Hypertens. 51: 69-76.

Hermida RC, Ayala DE, Portaluppi F (2007). Circadian variation of blood pressure: The basis for the chronotherapy of hypertension. Advanced Drug Delivery Reviews. 59(9-10): 904-22.

Horne JA, Ostberg O (1976). A self-assessment questionnaire to determine morningness-eveningness in human circadian rhythms. Int J Chronobiol. 4: 97-110.

Irigoyen MC, Consolim-Colombo FM, Krieger EM (2001). Controle cardiovascular: regulação reflexa e papel do sistema nervoso simpático. Rev Bras Hipertens. 8(1): 5562.

Jansen JM, Lopes AJ, Jansen U, Capone D, Maeda TY, Noronha A, Magalhães G, organizadores (2007). Medicina da noite: da cronobiologia à prática clínica. Ed. Fiocruz, Rio de Janeiro, Brasil, p. 210-54.

Kani AS, Sunbul M, Kani HT, Yanartas O, Tezcan N, Emul M (2015). Dream anxiety, chronotype and dipping pattern in hypertensive patients assessed with $24 \mathrm{~h}$ ambulatory blood pressure monitoring. Sleep Biol Rhythms. 14: 23-30.

Kidd PB, Young MW, Siggia ED (2015). Temperature compensation and temperature sensation in the circadian clock. Proc Natl Acad Sci USA. 112(46): 6284-92.

Knutson KL, Schantz M (2018). Associations between chronotype, morbidity and mortality in the UK Biobank cohort. Chronobiology International. 35(8): 1045-53.

Matchock RL (2018). Evening chronotype is associated with a more unrestricted sociosexuality in men and women. Personality and Individual Differences. 135(6): 56-9.

Mochizuki Y, Okutani M, Donfeng Y, Iwasaki H, Takusagawa M, Kohno I, et al (1998). Limited Reproducibility of Circadian Variation in Blood Pressure Dippers and Nondippers. J Hypertens. 11(4): 403-9.

Noll CA, Lee ENH, Schmidt A, Coelho EB, Nobre F (2001). Ausência de queda da pressão arterial entre os períodos de vigília e sono. Rev Bras Hipertens. 8(4): 468-72.

O’Brien E, Sheridan J, O’Malley K (1988). Dippers and Nondippers. The Lancet. 332(8607): 397.

Ohkubo T, Hozawa A, Yamaguchi J, Kikuya M, Ohmori K, Michimata M, et al (2002). Prognostic significance of the nocturnal decline in blood pressure in individuals with and without high 24-h blood pressure: the Ohasama study. J Hypertension. 77(2): 354-67.

Paine SJ, Gander PH, Travier N (2006). The epidemiology of morningness/eveningness: Influence of age, gender, ethnicity, and socioeconomic factors in adults (30-49 years). Journal of Biological Rhythms. 21(1): 68-76.

Pereira DS, Tufik S, Pedrazzoli M (2009). Moléculas que marcam o tempo: implicações para os fenótipos circadianos. Rev. Bras. Psiquiatr. 31(1): 63-71. 
Pickering TG (1990). The Clinical Significance of Diurnal Blood Pressure Variations - Editorial Comment. Circulation. 81(2): 700-3.

Pirsarabi F, Aghaali M, Rezvan S, Kashani SH (2018). Investigation of the Circadian Rhythm of Blood Pressure in Patients with Metabolic Syndrome and its Association with Metabolic Parameters. Qom Univ Med Sci J. 12(1): $72-9$.

Roenneberg T, Wirz-Justice A, Merrow M (2003). Life between clocks: Daily temporal patterns of human chronotypes. J Biological Rhythms. 18(1): 80-90.

Roman MJ, Pickering TG, Schwartz JE, Cavallini MC, Pini R, Devereux RB (1997). Is the absence of a normal nocturnal fall in blood pressure (nondipping) associated with cardiovascular target organ damage? J Hypertens. 15: 969-78.

Sierra A, Redon J, Banegas R, Parati G, Gorostidi M, Cruz JJ, et al (2009). Prevalence and Factors Associated With Circadian Blood Pressure Patterns in Hypertensive Patients. J Hypertens. 53: 466-72.
Taillard J, Philip P, Coste O, Sagaspe P, Bioulac B (2003). The circadian and homeostatic modulation of sleep pressure during wakefulness differs between morning and evening chronotypes. J Sleep Research. 12(4): 275-82.

Tobaldini E, Fiorelli EM, Solbiati M, Costantino G, Nobili L, Montano N (2019). Short sleep duration and cardiometabolic risk: from pathophysiology to clinical evidence. Nature Reviews Cardiology. 16(4): 213-24.

Verdecchia P, Schillaci G, Gatteschi C, Zampi I, Battistelli M, Bartoccini C, Porcellati C (1993). Blunted nocturnal fall in blood pressure in hypertensive women with future cardiovascular morbid events. Circulation. 88(3): 986-92.

Volkov GA, Baker KC, Foster J, Clemmons J, Jovanov E, Markin VS (2011). Circadian variations in biologically closed electrochemical circuits in Aloe vera and Mimosa pudica. Bioelectrochemistry. 81: 39-45. 